\title{
Urogenital Sinus
}

National Cancer Institute

\section{Source}

National Cancer Institute. Urogenital Sinus. NCI Thesaurus. Code C34322.

The ventral compartment of the cloaca following its division from the urogenital septum; it will ultimately form the majority of the bladder, the entire urethra in females, part of the urethra in males, and the vestibule of the vagina in females. 